\title{
Regulatory Focus and Self-Construal as Determinants of the Majority Rule in Individual Decision Making
}

\author{
Yong Lu, Marek Nieznański \\ Institute of Psychology, Faculty of Christian Philosophy \\ Cardinal Stefan Wyszyński University in Warsaw
}

\begin{abstract}
The majority rule has attracted much attention in recent debate on preference for aggregation strategies, which individuals may use in making choices or judgments for binary, weak-dominant multi-attribute options. The present research extends previous work on factors determining application of the majority rule. The experiment evidenced that individual decision makers are more likely to use the majority rule when increasing their orientation toward prevention rather than promotion regulatory focus. The results also confirmed a certain favorable tendency for individual decision makers to comply with the majority rule when priming their preference for interdependent rather than independent self-construal. These findings highlight a dynamic association between individual differences in goal pursuit motivation and perceived self-definition and behavioral judgment strategies.
\end{abstract}

Key words: the majority rule, regulatory focus, self-construal, binary choice

\section{Introduction}

Zhang, Hsee, and Xiao (2006) (see also Birnbaum \& Diecidue, 2015) examined a behavioral decision heuristic named the majority rule for choice between binary, weak-dominant multiattribute options in individual decision making. The majority rule posits that individuals prefer to choose the majority-weakly-superior option (i.e., slightly more favorable on most of its at- tributes) rather than the minority-strongly-superior option (i.e., considerably more favorable on few of its attributes; A brief formal description of the majority rule is provided in the Appendix; cf., May, 1952 for the pioneering axiomatic characterization of the majority rule). This preference could have stemmed from a deepseated belief that a congruent decision is more effective at receiving accurate information than an individual's effort (e.g., Hastie \& Kameda, 2005), or that the majoritarian judgments are,

Acknowledgements: We thank Prof. Dr. Li-Qiu Liu for conducting the experiment and thank Prof. Dr. Jiao Zhang, Prof. Dr. Andreas Glöckner, and two anonymous referees for extremely helpful written comments on the content herein. The paper also benefited from discussions with our colleagues Ms. Patrycja Wyszyńska, Mr. Michał Obidziński, and Ms. Eliza Smakuszewska.

Portions of an earlier first section of this paper were reported at the 2016 Conference (September 5 - 10) of Poznań Reasoning Week, Poznań, Poland (see Lu, 2016a).

Correspondence concerning this article should be addressed to Yong Lu or Marek Nieznański, Institute of Psychology, Faculty of Christian Philosophy, Cardinal Stefan Wyszyński University in Warsaw, ul. Wóycickiego 1/3, No. 14 Building, Warsaw 01-938, Poland, E-mail: luyong@student.uksw.edu.pl; yong.lu@student.kuleuven.be; m.nieznanski@uksw.edu.pl

Received April 18, 2017 
for instance, more democratic and just than the assertive judgments of the minority for satisfying political equality, with most elections of legislative representatives and referendums being decided by this rule (e.g., Nieuwelink, Dekker, Geijsel, \& Ten Dam, 2017; Risse, 2004; Saunders, 2008, p. 21).

A growing body of social choice literature has investigated the effect of the majority rule on the outcomes of a group choice process, both theoretically and experimentally. Dasgupta and Maskin (2008) argued that the majority rule is theoretically satisfactory to several appealing conditions over a larger cluster of preferences than any other voting rule. Hastie and Kameda (2005) proposed that the majority rule is popularly adopted among other eight truthseeking group decision rules, faring much better than the individual judgment averaging rule. Kimura and Katayama (2013) indicated, through a neurocognitive mechanism study, that the majority rule modulates the evaluative processing of monetary losses and the evaluation of conflicts among individual opinions in a threeparticipant group. The majority rule is also examined as a pivotal moderating factor, which influences the relationship between intra-team process conflict and cognitive diversity, namely rational and spontaneous styles (Fitzgerald, Mohammed, \& Kremer, 2017), and between social diversity and the efficacy of group decision making (McGrimmon, 2011). Compared with another commonly used group decision method of social choice, the unanimity rule (rule by consensus), the majority rule shows an advantage in arriving at a quicker decision (Taylor, Hewitt, Reeves, Hobbs, \& Lawless, 2013), indicates a better performance accuracy (Sorkin, West, \& Robinson, 1998), counteracts the negative effects of egoistic motivation on joint outcomes in asymmetrical negotiations (Beersma \& De Dreu, 2002), results in different decisions when group members are informed of one another's preference for the options (Miller, 1985), leads to weaker communication effects (Baillon, Bleichrodt, Liu, \& Wakker, 2016), and achieves less decision implementation and outcome satisfaction (Marsden \& Mathiyalakan, 2003; Mohammed \& Ringseis, 2001). Overall, previous work from a scattering of empirical and anecdotal sources provided credible evidence in support of the majority rule as a pervasively robust norm in group judgment and decision making.

While many studies have focused on the merits of the application of the majority rule in group decisions, only a handful of studies have examined its implications in individual decision making. For example, Russo and Dosher (1983) provided direct evidence supporting the use of the majority rule against the minority or neutral rule. May (1954) and Paterson and Diekmann (1988 found that their respondents might have used the majority rule but also violated transitivity axiom (i.e., if $X \rightarrow Y$ and $Y \rightarrow Z$, then $X \rightarrow$ $Z$ ) and the expected utility theory. However, Birnbaum and Diecidue (2015) argued against Zhang et al.'s (2006) proposition of the majority rule as a description of how individuals make a choice in that the majority of their participants not only dissatisfied the majority rule, but also simultaneously violated risk aversion and the transitivity and dominance axioms. In this sense, the so-far scarce studies have shown contradictory evidence concerning the potentially pivotal effects of the majority rule in individual decision making.

To date, only a limited number of research studies, to our knowledge, has filled the void to find a certain number of determinants that influence the application of the majority rule and, therefore, have specified some of the crucial conditions under which individuals choose to use it. Those determinants include such variables as number of attributes (less vs. more), information presentation format (choice vs. rating; attribute vs. option), attribute-unpacking (one attribute vs. several sub-attributes), and 
attribute-regrouping (lower-layer vs. higherlayer). Russo and Dosher (1983) showed that including higher numbers of attributes in binary, multi-attribute options may result in more relying on the majority rule. Zhang et al. (2006) found that individuals are more apt to use the majority rule when 1) the response mode is presented in choice rather than in rating, 2) the information format is presented in favor of intra-attribute comparison, namely comparing two options on one attribute after another, rather than intra-option integration, namely taking all the attributes of an option into account to form an integrate evaluation of the option, 3) a dominant attribute (e.g., English skills) is unpacked into a number of sub-attributes (e.g., spoken English skills and written English skills), and 4) the lower-layer attributes (e.g., ratings on two laptops' several features) rather than the higher-layer attributes (e.g., each of the features) are applicable to differentiate the majority-weakly-superior option. Nevertheless, it still opens a question about the other underlying factors that may cause systematic differences as to the use of the majority rule. In the present study, we aim to examine the roles of regulatory focus and self-construal that may account for decision makers to use the majority rule as a heuristic strategy when choosing an optimal alternative between weak dominant pairwise options.

Regulatory focus theory proposes two distinctly motivational states for all goal-directed behavior: prevention and promotion (Higgins, 2008; Higgins, Friedman, Harlow, Idson, Ayduk, \& Taylor, 2001; Higgins, Roney, Crowe, \& Hymes, 1994). In a prevention focus, individuals prefer a defensive strategy, which leads to error and loss avoidance (security and safety), a high sensitivity to negative events, and the fulfillment of obligations, whereas in a promotion focus, individuals prefer an eager strategy, which leads to seeking any specific goal (e.g., the pursuit of gains and aspiration toward ide- als and hedonic pleasure) and a particular sensitivity to positive information (cf., Crowe \& Higgins, 1997; Gino \& Margolis, 2011; Uskul, Sherman, \& Fitzgibbon, 2009). A burgeoning literature has demonstrated the impact of these differently motivational focuses on such phenomena as behavioral strategies (e.g., Kark, Katz-Navon, \& Delegach, 2015; Wang \& Lee, 2006) and risky information-processing style (e.g., Aaker \& Lee, 2001; Förster, Higgins, \& Bianco, 2003). Moreover, the majority rule can be assumed as a kind of defensive strategy, since the achievement of arriving at a majoritarian congruent agreement may involve the provision of support to, for example, compromise in order to fulfill certain obligations (e.g., group harmony, incoherence objection, solidarity), whereas "compromise [per se] can also be understood as a consequentialist justification of majority rule" (Risse, 2004, p. 45). Besides, Kimura and Katayama (2013) found direct evidence showing that monetary losses elicit more significant amplitude of outcome evaluation when modulated under the majority rule condition than under the unanimity rule and minority rule conditions, which indicated that using the majority rule evokes more attention to loss avoidance and negative events than using the other two rules. In addition, previous studies have also shown that emphasis on the descriptions of avoiding risk and loss and of achieving profit can temporarily evoke accessibility of prevention and promotion focuses, respectively (e.g., Bandura, 1991; Crowe \& Higgins, 1997; Gino \& Margolis, 2011; Higgins, 2008; Sassenberg, Sassenrath, \& Fetterman, 2015). Taken together, given the potential effect of the prevention focus on choosing to use the majority rule, we propose the following hypothesis:

Hypothesis 1. Individuals in the prevention focus condition are more likely to use the majority rule than individuals in the promotion focus condition. 
Accumulative research also suggests that self-construal impacts human perception and cognition in a various ways, including for example, relational and well-being assessment (Cross \& Morris, 2003), changes in visual scenes (Choi, Connor, Wason, \& Kahan, 2016), organizational events (Pekerti \& Kwantes, 2011), and opinion formation (e.g., Ybarra \& Trafimow, 1998) in which the use of a subjective heuristic (e.g., the majority rule) may be involved. Selfconstrual refers to views of self and how a person defines the self in relation to others. Markus and Kitayama (1991) distinguished between interdependent self-construal, which emphasizes a social relationship orientation and associated values, and independent self-construal, which is associated with individual attributes and autonomy (see Cross, Hardin, \& GercekSwing, 2011 for an overview). Specifically, interdependent self-construal may be more consistent with the assumption of the majority rule, since the goal of interdependent self-construal seeks to conform to the majoritarian agreement, with an emphasis on maintaining such values as collectivism (e.g., Lu \& Gilmour, 2007), common humanity (e.g., Akin \& Erolu, 2013; Markus \& Kitayama, 1991), connectedness (e.g., Singelis, 1994), group loyalty (e.g., Fernández, Paez, \& González, 2005), holistic thinking (e.g., Markus \& Kitayama, 1991), and relatedness to others (e.g., Avsec, 2003; Cross, Bacon, \& Morris, 2000). Therefore, given the potential influence of the interdependent self-construal on choosing to use the majority rule, we propose the following hypothesis:

Hypothesis 2. Priming the interdependent selfconstrual causes individuals more likely to use the majority rule in comparison with priming the independent self-construal.

In the present study, we focus on the weak dominant binary choices in the situation under uncertainty and investigate whether different levels of regulatory focus and self-construal induce people choosing to use the majority rule.
Next, we test the two hypotheses through one experiment.

\section{Method}

\section{Participants}

A total of 107 undergraduate students who specialized in Business Administration, Engineering Management, Finance, or Logistics Engineering in Tianjin University, China volunteered to take part in the study. The age of the participants ranged from 18 to 23 years old $(M=$ $19.96, S D=.95)$. The female percentage of the participants was $35.5 \%$. Participants did not receive financial compensation since the task took only a few minutes to complete. Nevertheless, participants received an extra course credit in exchange for participating in the experiment.

\section{Design and Materials}

We presented to the participants a simulated vignette concerning credit ratings made by four institutions $A, B, C$, and $D$ on two investment corporations $X$ and $Y$. Since placing an emphasis on avoiding loss and achieving profitability can temporarily evoke accessibility of correspondingly prevention and promotion focuses, we evoked participants' regulatory focuses by priming the stimulus' description either focusing on the four institutions' credit ratings on loss avoidance or benefit gain. Moreover, in order to evoke participants' two particular selfconstruals, we asked them to think of either their own or family deposit to invest. Thus, a 2 (situational framing of loss avoidance vs. profit gain - that is, prevention focus vs. promotion focus) $\times 2$ (family deposit vs. individual deposit that is, interdependent self-construal vs. independent self-construal) between-subjects design was used. Participants were randomly assigned to one of the four cells. In this choice task, all of the participants first read: 
There are two Corporations, X and Y, who provide risk investment products for their clients.

The choice problem between the two corporations were displayed using the format shown in Table 1 (under the prevention focus condition) or Table 2 (under the promotion focus condition), where the numbers underneath $A, B, C$, and $D$ represented ratings of the four credit rating institutions. The order of the four institutions' ratings was counterbalanced. We constructed the average ratings to be the same; therefore, some people who normally use mean ratings might use the majority rule as a secondary strategy when the means are equal. Half of the participants (another half) read the following prevention-framed (promotion-framed) description related to the four institutions' loss (benefit) ratings:

There are four Credit Rating Institutions, A, B, C, and D, who have affirmed X's and Y's loss (profit) ratings at the following table.

The scenario which evokes the interdependent (independent) self-construal condition reads:

Suppose your family (you) plan to withdraw part of your family's (your) deposit to invest in alternative one of the corporations' risk invest-

Table 1 The four institutions' loss ratings on the corporations $X$ and $Y$

\begin{tabular}{ccccc}
\hline Loss Ratings $^{\text {a }}$ & $A$ & $B$ & $C$ & $D$ \\
\hline$X$ & 2 & 5 & 2 & 3 \\
$Y$ & 3 & 1 & 4 & 4 \\
\hline
\end{tabular}

${ }^{\mathrm{a}}$ On a 11-point scale, ranging from 0 (no loss) to 10 (maximum loss)

Table 2 The four institutions' profit ratings on the corporations $X$ and $Y$

\begin{tabular}{ccccc}
\hline Profit Ratings $^{\mathrm{a}}$ & $A$ & $B$ & $C$ & $D$ \\
\hline$X$ & 8 & 5 & 8 & 7 \\
$Y$ & 7 & 9 & 6 & 6 \\
\hline
\end{tabular}

${ }^{\mathrm{a}}$ On a 11-point scale, ranging from 0 (no profit) to 10 (maximum profit) ment products. What is your family's (your) choice?

In order to check the effectiveness of manipulation of interdependent and independent selfconstruals, we followed Aaker and Lee's (2001) method by asking participants:

While you were reading about the abovementioned scenario and question, please describe the extent to which 1) you thought just about yourself, 2) your thoughts about the message were focused on just yourself, 3) your thoughts were focused on just you, 4) you thought about you and your family, 5) your thoughts about the message were focused on you and your family, and 6) your thoughts were focused on you and your family.

Participants responded on a seven-point Likert scale ( $1=$ not at all; $7=\mathrm{a}$ lot $)$. The first three items are averaged to form a Self Thoughts Index, and the remaining three items are averaged to form an Others Thoughts Index.

\section{Procedure}

The experiment was conducted in one quiet classroom. As the study presented no more than minimal risk of harm to participants and involved no procedures for which written consent is normally required outside of the study context, the Ethics Committee specifically approved verbal informed consent being provided to participants in lieu of signed informed consent. The experimenter read out loud the verbal informed consent at the outset of the experiment. All of participants consented to participate. After that, the experimenter gave each participant one leaflet containing the questions, and participants answered individually at their seats.

\section{Results and Discussion}

We excluded 5 participants who did not choose either $X$ or $Y$ and another 1 participant 
who did not give her ratings to the Self Thoughts Index and the Others Thoughts Index from the analyses. We used an alpha level of .05 for all significance tests.

For the remaining 101 participants, Cronbach's $\alpha$ for the Self Thoughts Index and the Others Thoughts Index were .878 and .797, respectively. The participants in the interdependent self-construal condition thought more about the self with their family $(M=5.511, S D=$ .017) than the individual $\operatorname{self}(M=3.532, S D=$ $.266 ; t=4.701, p<.001)$. The participants in the independent self-construal condition thought more about the individual self $(M=6.716, S D=$ $.004)$ than the self with their family $(M=1.411$, $S D=.066 ; t=21.509, p<.001)$. The results provided evidence that the manipulation primed interdependent and independent self-construals effectively.

Response frequencies and percentages of participants' choice under different experimental conditions are shown in Table 3. Participants who preferred the corporation that was judged better by a majority of the four credit rating institutions were regarded as using the strategy of the majority rule; otherwise, they were treated as using the "other" strategies. On the one hand, in the prevention focus condition $(N=$ 47), $83.0 \%$ and $17.0 \%$ participants' choices were in accordance with respectively the majority rule and any other strategies, which was signifi- cantly different compared with those of $55.6 \%$ and $44.4 \%$ participants' choices in the promotion focus condition $(N=54)\left(\chi^{2}[1]=8.731, p=\right.$ .005 , Cohen's $d=.616)$. Following Cohen's guideline $(d=.20, .50$, and .80 for small, medium, and large effects, respectively; cf., Cohen, 1992), the difference between the means of the two compared groups for their choices was a medium effect. Participants' choices in the prevention focus condition were more likely to comply with the majority rule than in the promotion focus condition. Therefore, Hypothesis 1 was confirmed.

On the other hand, in the interdependent selfconstrual condition $(N=51), 76.5 \%$ and $23.5 \%$ participants' choices were in accordance with the majority rule and any other strategies, respectively, which just slightly missed the significance level of differences, compared with those of $40 \%$ and $60 \%$ participants' choices in the independent self-construal condition $(N=$ 50) $\left(\chi^{2}[1]=3.164, p=.090\right.$, Cohen's $\left.d=.356\right)$. The difference between the means of the two compared groups for their choices was a small effect. Thus, participants' choices in the interdependent self-construal condition showed a certain favorable tendency to be more likely to comply with the majority rule than in the independent self-construal condition. It is worth mentioning that Taylor et al. (2013) employed a self-report ratings ranging from a -1.0 (i.e., no

Table 3 Contingency table presenting participants' choices by experimental conditions

\begin{tabular}{|c|c|c|c|c|c|c|}
\hline \multirow[b]{3}{*}{ Strategies } & \multicolumn{6}{|c|}{ Conditions } \\
\hline & \multicolumn{2}{|c|}{ Prevention } & \multirow[b]{2}{*}{ Sum } & \multicolumn{2}{|c|}{ Promotion } & \multirow[b]{2}{*}{ Sum } \\
\hline & Interdependent & Independent & & Interdependent & Independent & \\
\hline $\begin{array}{l}\text { The } \\
\text { majority } \\
\text { rule }\end{array}$ & $\begin{array}{c}22 \\
(88.0 \%)\end{array}$ & $\begin{array}{c}17 \\
(77.3 \%)\end{array}$ & $\begin{array}{c}39 \\
(83.0 \%)\end{array}$ & $\begin{array}{c}17 \\
(65.4 \%)\end{array}$ & $\begin{array}{c}13 \\
(46.4 \%)\end{array}$ & $\begin{array}{c}30 \\
(55.6 \%)\end{array}$ \\
\hline Others & $\begin{array}{c}3 \\
(12.0 \%)\end{array}$ & $\begin{array}{c}5 \\
(22.7 \%)\end{array}$ & $\begin{array}{c}8 \\
(17.0 \%)\end{array}$ & $\begin{array}{c}9 \\
(34.6 \%)\end{array}$ & $\begin{array}{c}15 \\
(53.6 \%)\end{array}$ & $\begin{array}{c}24 \\
(44.4 \%)\end{array}$ \\
\hline$N$ & $\begin{array}{c}25 \\
(100 \%)\end{array}$ & $\begin{array}{c}22 \\
(100 \%)\end{array}$ & $\begin{array}{c}47 \\
(100 \%)\end{array}$ & $\begin{array}{c}26 \\
(100 \%)\end{array}$ & $\begin{array}{c}28 \\
(100 \%)\end{array}$ & $\begin{array}{c}54 \\
(100 \%)\end{array}$ \\
\hline
\end{tabular}


social effects and full independence among agents) up to +1.0 (i.e., no individual and only group effects) to analyze how their three to seven-person groups affected individuals' interdependence. Their results found a moderate amount of interdependence (0.375) across all groups, which indicated that the majority rule generates more interdependence than the consensus rule in seeking solutions to problems. Compared to their findings, we reversely manipulated the condition of self-construals to measure its impact on choosing to use the majority rule. Nonetheless, the combined results provide evidence to support a mutually positive correlation of the interdependent selfconstrual and the majority rule. Therefore, Hypothesis 2 was supported.

More specifically, on the one hand, since people are loss averse, as perceived by the asymmetrical value function of the prospect theory (Kahneman \& Tversky, 1979), the results can be explained by decreasing marginal returns (i.e., differences are perceived as smaller for larger numbers), in which the differences of the perceptions of outcomes for the loss/profit framing are driven by loss aversion. In other words, the participants in the prevention focus condition probably perceived larger differences of institutions' ratings, and thus, were more risk averse than those in the promotion focus condition. On the other hand, Pahlke, Strasser, and Vieider (2015) found that bearing responsibility for others' payoffs (an interdependent selfconstrual induction) as well as for oneself increases risk aversion for small losses and moderate to large gains under risk, whereas for large losses and small gains under risk, it increases risk seeking. Further, Girtz, Hill, and Owens (2017) found that having responsibility for another player in the Stag Hunt game amplifies risk-averse behavior. Although their research together with other literature on the extent to which responsibility accounts for risk preference in individual decision making may reach widely mixed conclusions (for a review, see Pahlke et al., 2015), we could still assume a certain causal relationship between taking responsibility for others and risk aversion. Taken together, both prevention focus and interdependent self-construal may lead to a conservative risk-aversion behavior, and according to Kimura and Katayama (2013), risk aversion, in turn, results in a more favorite preference of the majority rule.

Furthermore, a generalized linear model with a logit link function was run using IBM SPSS Package version 22 to test the interaction effects between the two dichotomous determinants, regulatory focus (prevention vs. promotion) and self-construal (interdependent vs. independent). The determinants were included in the model as predictors, while the participants' choices were used as binary dependent variable. The analysis showed, however, no significant interaction of the effects (Wald $\chi^{2}[1]=$ $0.000, S E=.975, p=.991$ ), which failed to provide evidence in support of a more general finding in previous research that individuals with a dominant interdependent self-construal tend to exhibit a bias toward prevention focus, whereas those with a dominant independent selfconstrual tend to exhibit a bias toward promotion focus (e.g., Aaker \& Lee, 2001; Elliot, Chirkov, Kim, \& Sheldon, 2001; Lee, Aaker, \& Gardner, 2000; Lin, Chang, \& Lin, 2012). Nonetheless, our results support the notion that regulatory focus is an orthogonal predictor in a manner consistent with the pattern of such findings, as that prevention focus sheds no moderation effect on fair pay dispersion (Park, Kim, \& Sung, 2017) and that controlling for the potential influence of regulatory focus has no significant main or interaction effects on expectation of goal completion (Yang, Stamatogiannakis, \& Chattopadhyay, 2015).

Additionally, it is important to realize that since we employed just a single choice problem which could confound serial position, our find- 
ings are also consistent with a summing strategy, such as the weighted average rule, in that there are weights for serial positions (e.g., a participant assigned lower weight to $B$ 's ratings in Table 1 or 2) that the participant could also prefer $X$ to $Y$ or $Y$ to $X$ based on a weighted average.

\section{Conclusions}

The majority rule could be regarded as one of the dimensional strategies judged based on attributes across options, which, according to the perspective of simple heuristic conceptual approaches (e.g., Martignon \& Hoffrage, 2002), is more preferred by individuals than holistic strategies judged based on an option (e.g., expected utility calculation) for multi-attribute binary choice (Jansen, van Duijvenvoorde, \& Huizenga, 2012; Russo \& Dosher, 1983). Although this ecological adaptation of decision making may simplify their mathematical computations of the problem and may eliminate their cognitive loads, it also unavoidably leads to systematic violations of the expected utility theory on certain choices. Nevertheless, these elaborate strategies suggest adaptive implications for judgment under uncertainty.

It is noteworthy that the priority heuristic (Brandstätter, Gigerenzer, \& Hertwig, 2006) can be regarded as an advanced adaptation of the majority rule (also including a cluster of other similar heuristics such as the max-min rule, the min-max rule, the maximum rule) in that this heuristic models the actual majority behavior and especially defines certain conditions as the corresponding judgmental criteria (e.g., maximum gains first). Another heuristic strategy called the equate-to-differentiate rule, which states that decision makers first regard one or several smaller different attributes of options as equivalent to each other and then leave the most distinct attribute as the determinant of the final pairwise choice (Li, 2004). The main hypothesis of this rule is probably based on approximate estimation (Chesney, Bjalkebring, \& Peters, 2015) or on a philosophical perspective, which emphasizes the dominant minority's rights in a group in consideration of racial (or other deep) divisions (Guinier, 1994, p. 9). Although the majority rule and the equate-to-differentiate rule share a close decision process by, in case of pairwise choice, detecting dominant distinctions between different attributes of options, the two rules come to contradictory decision results; that is, the equate-to-differentiate rule postulates that individuals will choose the minority-strongly-superior option.

Like the majority rule which shows its robust beauty in group decisions (for an elaborate review, see Hastie \& Kameda, 2005), the priority heuristic and the equate-to-differentiate rule also perform successfully on various inference procedures or in a series of competitions with other modifications of the expected utility theory (e.g., the cumulative prospect theory) which, on the other hand, account for the same data poorly (e.g., Brandstatter et al., 2006; Li, 2016). From an adaptive, evolutionary point of view, those behavioral heuristics are among others the kind of "rules of thumb" which represent fast and frugal judgmental process, whereby people could capture the asymmetry for evaluations of, for instance, gains and losses across different dimensions among options. Further, those heuristics assume human choice behavior as a process of seeking a significant difference between options either on the majoritarian dimensions or on only one dimension with a primary priority or the greatest dimensional difference. Although adequate evidence has shown that human choice under risk and uncertainty is heterogeneous, a favorite opinion also supports the argument that people incorrectly use certain compensatory strategies (e.g., the average rule, the expected utility theory) or non-compensatory strategies (e.g., the majority rule, the priority rule, the equate-to-dif- 
ferentiate rule) as their "rational" solutions, which has steadily challenged the traditional computations of accurate "summing and weighing" by simplifying and modelling step by step heuristic considerations that require less cognitive effort (for a recent review, see Lu, 2016b).

The present study showed that the participants' judgments were congruent with the prediction of the majority rule by manipulations that increased their prevention focus or primed their interdependent self-construal. Primingbased induction can be interpreted as impacting what is constructed as salient at the moment of judgment, and more chronic sources of construct accessibility (e.g., risk preference) are important to judgmental formation as well (Bargh, Bond, Lombardi, \& Tota, 1986). On the one hand, results from Motyka, Grewal, Puccinelli, Roggeveen, Avnet, Daryanto et al.'s (2014) meta-analysis indicated that prevention focus yields a stronger effect on evaluation compared to promotion focus, whereas promotion focus has a strong effect on behavior in comparison with prevention focus. However, prevention and promotion focuses yield no difference in strength of effects for behavioral intention. Kimura and Katayama's (2013) electrophysiological study results support the notion that risk-aversive-related outcome evaluation is associated with using the majority rule in a manner consistent with the present findings. On the other hand, previous studies have focused on whether self-construal as an individual difference construct plays a vital role in, for example, potential biases resulting from judgmental heuristics (e.g., Bry, Follenfant, \& Meyer, 2008; Cheek \& Norem, 2017) and in mediating social emotions (e.g., Hui, Fok, \& Bond, 2009; Levinson, Langer, \& Rodebaugh, 2011; Lun, Kesebir, \& Oishi, 2008) and consumer behaviors (e.g., Chen, 2009; Wang \& Keh, 2017). The present study extends previous work on the role of self-construal in the scope of individual decision making.
The current study also has a number of limitations. First, the possible value of the paper may not lie in the scope of comparing the majority rule with these choice strategies that would predict the opposite results (cf., Baillon et al., 2016; Hastie \& Kameda, 2005; Miller, 1985; Sorkin et al., 1998; Taylor et al., 2013) or with these compatible strategies (e.g., the additive or equal-weighted rule); rather, we aim to show that certain situations facilitate the use of the majority rule. Second, different from these experimental designs with repeatedly consequential decisions between options, which enabled to test the coherence of behavior according to axiomatic criteria when using the majority rule (e.g., Birnbaum \& Diecidue, 2015), we applied a single choice task, which failed to examine any refutation of such axioms (e.g., the transitivity axiom) and to consider the effect of random errors on the choice results. Third, although we used an experimental design to prime prevention versus promotion self-construal, evidence suggests that self-construal can still substantially vary at the individual level within cultural groups (Han \& Humphreys, 2016; Oyserman, Coon, \& Kemmelmeier, 2002). However, the participants did not differ in their racial/ethnic composition, and thus, each condition should be presumed as being equally impacted by the cultural influence. Furthermore, for manipulation check purposes, we followed prior work by assessing self-construals unidimensionally (e.g., Aaker \& Lee, 2001), and the observed effects were statistically attributable to our manipulation. However, such an approach is likely an oversimplification of self-construal (Cross et al., 2011) as well as regulatory focus. Nevertheless, we abandoned such most commonly used instruments as the Regulatory Focus Questionnaire (Higgins et al., 2001) and the Independent and Interdependent Self-Construal Scales (Singelis, 1994), since their validity has been heavily questioned (e.g., Levine, Bresnahan, Park, Lapinski, Wittenbaum, 
Shearman et al., 2003; Summerville \& Roese, 2008).

Several directions of research should be investigated in the future. The first direction may address specific hypotheses about factors that account for whether people use the majority rule or other distinctive decision strategies. The second direction concerns the effect of another possible difference between dimensions and dimension validity weights (i.e., conditional probabilities) on choosing to use the majority rule, which has not been explored but was just mentioned in the general discussion section of Zhang et al. (2006). The third research area may concern cross-cultural differences in the use of the majority rule, as we take into account that a well-established finding in the literature on culture is that, relatively speaking, people from Western and Eastern cultures are respectively individualistic and collectivistic (e.g., Gaechter, Herrmann, \& Thoni, 2005; Oeberst \& Wu, 2015). The fourth orientation may measure how the differences of individualism and collectivism (e.g., Jackson, Colquitt, Wesson, \& ZapataPhelan, 2006; Oyserman et al., 2002; Shavitt, Torelli, \& Riemer, 2011) influence the use of the majority rule in individual or group decision performance. The fifth direction may further examine the impact of the priming tasks on regulatory focus and self-construal by using a multidimensional instead of a unidimensional approach. At this point, future research should continue to examine the measurement properties of these constructs. The sixth direction concerns further examining regulatory focus as a determinant of the majority rule by means of differentiating evaluation and behavior purposes in consideration of the different effects of a prevention versus promotion focus on evaluation and behavior (Motyka et al., 2014).

The present contribution has a number of implications for both theory and practice in individual decision making and offers possible solutions to group thinking. Foremost, com- pared with previous work on the unpacking and order effects on the application of the majority rule, our results provide direct evidence for other two determinants, which are less dependent on the task options per se (e.g., attribute numbers, presentation format) and are more determined by the decision makers' characteristics (e.g., goal pursuit orientation) or by external environments (e.g., cultural differences). Concretely, the results indicated that prevention focus and interdependent self-construal show promise as two predictors for inducing people to use the majority rule. In practice, politicians may intend to choose people with interdependent characteristics or to specify prevention/vigilant decision-making environment in order to enhance the majoritarian congruent judgments or to influence the minority's decision. Nevertheless, there is a threat of unethical manipulations that prime people's regulatory focus or selfconstrual.

In summary, we believe that as a pervasive form of judgmental norm used most often in group as well as individual decision making, the majority rule can be better understood by incorporating other critically deterministic elements. Bearing this in mind, the goal of the present research was to explore the roles of regulatory focuses and self-construals for the preference of the majority rule in multi-attribute decisions. Our results drew a convergent picture: Individuals who are primed with the prevention focus are prone to use the majority rule. We also indicated a certain favorable trend for the majority rule application in the interdependent self-construal condition in comparison with the independent self-construal condition. Additionally, contrary to previous findings that regulatory focus seems to mediate the relationship between self-construal and decision making, no interaction effect was found between regulatory focus and self-construal. These study results augmented the influence of deterministic factors on choosing to use the majority rule 
and showed promise for continued investigation of determination or mediation factors for the majority rule at the individual level.

\section{References}

Aaker, J. L., \& Lee, A. Y. (2001). "I" seek pleasures and "we" avoid pains: The role of self-regulatory goals in information processing and persuasion. Journal of Consumer Research, 28, 33-49. doi:10.1086/ 321946

Akin, A., \& Erolu, Y. (2013). Self-compassion and relational-interdependent self-construal. Studia Psychologica, 55, 111-121. doi:10.21909/sp.2013. 02.629

Avsec, A. (2003). Masculinity and femininity personality traits and self-construal. Studia Psychologica, 45, 151-159.

Baillon, A., Bleichrodt, H., Liu, N., \& Wakker, P. P. (2016). Group decision rules and group rationality under risk. Journal of Risk and Uncertainty, 52, 99116. doi:10.1007/s11166-016-9237-8

Bandura, A. (1991). Social cognitive theory of selfregulation. Organizational Behavior and Human Decision Process, 50, 248-287. doi:10.1016/07495978(91)90022-1

Bargh, J. A., Bond, R. N., Lombardi, W. J., \& Tota, M E. (1986). The additive nature of chronic and temporary sources of construct accessibility. Journal of Personality and Social Psychology, 50, 869-878. doi:10.1037//0022-3514.50.5.869

Beersma, B., \& De Dreu, C. K. W. (2002). Integrative and distributive negotiation in small groups: Effects of task structure, decision rule, and social motive. Organizational Behavior and Human Decision Processes, 87, 227-252. doi:10.1006/obhd.2001.2964

Birnbaum, M. H., \& Diecidue, E. (2015). Testing a class of models that includes majority rule and regret theories: Transitivity, recycling, and restricted branch independence. Decision, 2, 145-190. doi:10. $1037 /$ dec0000031

Brandstätter, E., Gigerenzer, G., \& Hertwig, R. (2006) The priority heuristic: Making choices without tradeoffs. Psychological Review, 133, 409-432. doi:10. 1037/0033-295x.113.2.409

Bry, C., Follenfant, A., \& Meyer, T. (2008). Blonde like me: When self-construals moderate stereotype priming effects on intellectual performance. Journal of Experimental Social Psychology, 44, 751757. doi:10.1016/j.jesp.2007.06.005

Cheek, N. N., \& Norem, J. K. (2017). Holistic thinkers anchor less: Exploring the roles of self-construal and thinking styles in anchoring susceptibility. Per- sonality and Individual Differences, 115, 174-176. doi:10.1016/j.paid.2016.01.034

Chen, C. Y. (2009). Who I am and how I think: The impact of self-construal on the roles of internal and external reference prices in price evaluations. Journal of Consumer Psychology, 19, 416-426. doi:10. 1016/j.jcps.2009.05.012

Chesney, D., Bjalkebring, P., \& Peters, E. (2015). How to estimate how well people estimate: Evaluating measures of individual differences in the approximate number system. Attention, Perception, \& Psychophysics, 77, 2781-2802. doi:10.3758/s13414015-0974-6

Choi, H., Connor, C. B., Wason, S. E., \& Kahan, T. A. (2016). The effects of interdependent and independent priming on Western participants' ability to perceive changes in visual scenes. Journal of CrossCultural Psychology, 47, 97-108. doi:10.1177/ 0022022115605384

Cohen, J. (1992). A power primer. Psychological Bulletin, 112, 155-159. doi:10.1037/0033-2909.112. 1.155

Cross, S. E., Bacon, P. L., \& Morris, M. L. (2000). The relational-interdependent self-construal and relationships. Journal of Personality and Social Psychology, 78, 791-808. doi:10.1037/0022-3514.78.4.791

Cross, S. E., Hardin, E. E., \& Gercek-Swing, B. (2011). The what, how, why, and where of self-construal. Personality and Social Psychology Review, 15, 142179. doi:10.1177/1088868310373752

Cross, S. E., \& Morris, M. L. (2003). Getting to know you: The relational self-construal, relational cognition, and well-being. Personality and Social Psychology Bulletin, 29, 512-523. doi:10.1177/ 0146167202250920

Crowe, E., \& Higgins, E. T. (1997). Regulatory focus and strategic inclinations: Promotion and prevention in decision making. Organizational Behavior and Human Decision Processes, 69, 117-132. doi:10.1006/obhd.1996.2675

Dasgupta, P., \& Maskin, E. (2008). On the robustness of majority rule. Journal of the European Economic Association, 6, 949-937. doi:10.1162/jeea.2008.6. 5.949

Elliot, A. J., Chirkov, V. I., Kim, Y., \& Sheldon, K. M. (2001). A cross-cultural analysis of avoidance (relative to approach) personal goals. Psychological Science, 12, 505-510. doi:10.1111/1467-9280.00393

Fernández, I., Paez, D., \& González, J. L. (2005). Independent and interdependent self-construals and socio-cultural factors in 29 nations. Revue Internationale de Psychologie Sociale, 18, 35-63.

Fitzgerald, D. R., Mohammed, S., \& Kremer, G. O. (2017). Differences in the way we decide: The effect of decision style diversity on process conflict in 
design teams. Personality and Individual Differences, 104, 339-344. doi:10.1016/j.paid.2016.08.030

Förster, J., Higgins, E. T., \& Bianco, A. T. (2003). Speed/accuracy decisions in task performance. Buildin trade-off or separate strategic concerns? Organizational Behavior and Human Decision Process, 90, 148-164. doi:10.1016/S0749-5978(02)005095

Gaechter, S., Herrmann, B., \& Thoni, C. (2005). Crosscultural differences in norm enforcement. Behavioral and Brian Sciences, 28, 822-823. doi:10.1017/ s0140525x05290143

Gino, F., \& Margolis, J. D. (2011). Bringing ethics into focus: How regulatory focus and risk preferences influence (un)ethical behavior. Organizational Behavior and Human Decision Processes, 115, 145 156. doi:10.1016/j.obhdp.2011.01.006

Girtz, R., Hill, J., \& Owens, M. (2017). Risk preferences, responsibility, and self-monitoring in a Stag Hunt. Journal of Behavioral and Experimental Economics, 68, 53-61. doi:10.1016/j.socec.2017.03.004

Guinier, L. (1994). The tyranny of the majority: Fundamental fairness in representative democracy. New York: Free Press.

Han, S., \& Humphreys, G. (2016). Self-construal: A cultural framework for brain function. Current Opinion in Psychology, 8, 10-14. doi:10.1016/j.copsyc. 2015.09.013

Hastie, R., \& Kameda, T. (2005). The robust beauty of majority rules in group decisions. Psychological Review, 112, 494-508. doi:10.1037/0033-295x.112. 2.494

Higgins, E. T. (2008). Promotion and prevention: Regulatory focus as a motivational principle. $A d$ vances in Experimental Social Psychology, 30, 146. doi:10.1016/S0065-2601(08)60381-0

Higgins, E. T., Friedman, R. S., Harlow, R. E., Idson, L. C., Ayduk, O. N., \& Taylor, A. (2001). Achievement orientations from subjective histories of success: Promotion pride versus prevention pride. European Journal of Social Psychology, 31, 3-23. doi:10.1002/ ejsp. 27

Higgins, E. T., Roney, C., Crowe, E., \& Hymes, C. (1994). Development of regulatory focus: Promotion and prevention as ways of living. In J. Heckhausen \& C. S. Dweck (Eds.), Motivation and self-regulation across the lifespan (pp. 50-77). New York: Cambridge University Press.

Hui, C. M., Fok, H. K., \& Bond, M. H. (2009). Who feels more ambivalence? Linking dialectical thinking to mixed emotions. Personality and Individual Differences, 46, 493-498. doi:10.1016/j.paid.2008. 11.022

Jackson, C. L., Colquitt, J. A., Wesson, M. J., \& ZapataPhelan, C. P. (2006). Psychological collectivism: A measurement validation and linkage to group member performance. Journal of Applied Psychology, 91, 884-899. doi:10.1037/0021-9010.91.4.884

Jansen, B. R. J., van Duijvenvoorde, A. C. K., \& Huizenga, H. M. (2012). Development of decision making: Sequential versus integrative rules. Journal of Experimental Child Psychology, 111, 87-100. doi:10.1016/j.jecp.2011.07.006

Kahneman, D., \& Tversky, A. (1979). Prospect theory: An analysis of decision under risk. Econometrica, 47, 263-292. doi:10.2307/1914185

Kark, R., Katz-Navon, T., \& Delegach, M. (2015). The dual effects of leading for safety: The mediating role of empolyee regulatory focus. Journal of Applied Psychology, 100, 1332-1348. doi:10.1037/ a0038818

Kimura, K., \& Katayama, J. (2013). Outcome evaluations in group decision making using the majority rule: An electrophysiological study. Psychophysiology, 50, 848-857. doi:10.1111/psyp. 12068

Lee, A. Y., Aaker, J. L., \& Gardner, W. L. (2000). The pleasures and pains of distinct self-construals: The role of interdependence in regulatory focus. Journal of Personality and Social Psychology, 78, 11221134. doi:10.1037/0022-3514.78.6.1122

Levine, T. R., Bresnahan, M. J., Park, H. S., Lapinski, M. K., Wittenbaum, G. M. W., Shearman, S. M. et al. (2003). Self-Construal Scales lack validity. $H u$ man Communication Research, 29, 210-252. doi:10.1111/j.1468-2958.2003.tb00837.x

Levinson, C. A., Langer, J. K., \& Rodebaugh, T. L. (2011). Self-construal and social anxiety: Considering personality. Personality and Individual Differences, 51, 355-359. doi:10.1016/j.paid.2011.04.006

Li, S. (2004). A behavior choice model when computational ability matters. Applied Intelligence, 20, 147163. doi: $10.1023 / \mathrm{b}$ :apin.0000013337.01711.c7

Li, S. (2016). An Equate-to-differentiate Way of Decision-making. Shanghai: East China Normal University Press.

Lin, Y. C., Chang, C. C. A., \& Lin, Y. F. (2012). Selfconstrual and regulatory focus influences on persuasion: The moderating role of perceived risk. Journal of Business Research, 65, 1152-1159. doi:10. 1016/j.jbusres.2011.08.001

Lu, L., \& Gilmour, R. (2007). Developing a new measure of independent and interdependent views of the self. Journal of Research in Personality, 41, $247-$ 257. doi:10.1016/j.jrp.2006.09.005

Lu, Y. (2016a). The majority rule or the equate-todifferentiate rule? The moderating role of regulatory focus, self-construals, and culture differences. In Abstracts of Poznań Reasoning Week 2016, pp. 24-26. https://poznanreasoningweek.files.wordpress. com/2016/09/prw2016abstracts.pdf 
Lu, Y. (2016b). The conjunction and disjunction fallacies: Explanations of the Linda problem by the equate-to-differentiate model. Integrative Psychological and Behavioral Science, 50, 507-531. doi:10.1007/s12124-015-9314-6

Lun, J., Kesebir, S., \& Oishi, S. (2008). On feeling understood and feeling well: The role of interdependence. Journal of Research in Personality, 42, 16231628. doi:10.1016/j.jrp.2008.06.009

Markus, H., \& Kitayama, S. (1991). Culture and the self: Implications for cognition, emotion, and motivation. Psychological Review, 98, 224-253. doi:10.1037/0033-295x.98.2.224

Marsden, J. R., \& Mathiyalakan, S. (2003). An investigation of changes in attitude over time of GDSS groups under unanimity and majority decision rules. European Journal of Operational Research, 145 , 693-712. doi:10.1016/s0377-2217(02)00224-2

Martignon, L., \& Hoffrage, U. (2002). Fast, frugal, and fit: Simple heuristics for paired comparison. Theory and Decision, 52, 29-71. doi:10.1023/ a:1015516217425

May, K. O. (1952). A set of independent necessary and sufficient conditions for simple majority decision. Econometrica, 20, 680-684. doi:0012-9682 (195210)20:4<680:asoina $>2.0 . \mathrm{co} ; 2-\mathrm{s}$

May, K. O. (1954). Intransitivity, utility, and the aggregation of preference patterns. Econometrica, 22, 1-13. doi:10.2307/1909827

McGrimmon, T. S. (2011). Social diversity, influence and the efficacy of majority rule: A theoretical, experimental and field investigation (Doctoral dissertation). University of South Carolina.

Miller, C. E. (1985). Group decision making under majority and unanimity decision rules. Social Psychology Quarterly, 48, 51-61. doi:10.2307/ 3033781

Mohammed, S., \& Ringseis, E. (2001). Cognitive diversity and consensus in group decision making: The role of inputs, processes, and outcomes. Organizational Behavior and Human Decision Processes, 85, 310-335. doi:10.1006/obhd.2000.2943

Motyka, S., Grewal, D., Puccinelli, N. M., Roggeveen, A. L., Avnet, T., Daryanto, A. et al. (2014). Regulatory fit: A meta-analytic synthesis. Journal of Consumer Psychology, 24, 294-410. doi:10.1016/ j.jcps.2013.11.004

Nieuwelink, H., Dekker, P., Geijsel, F., \& Ten Dam, G. (2017). "Democracy always comes first": Adolescents' views on decision-making in everyday life and political democracy. Journal of Youth Studies, 19, 990-1006. doi:10.1080/13676261.2015. 1136053 .

Oeberst, A., \& Wu, S. (2015). Independent vs. interdependent self-construal and interrogative compliance:
Intra- and cross-cultural evidence. Personality and Individual Differences, 85, 50-55. doi:10.1016/ j.paid.2015.04.038

Oyserman, D., Coon, H. M., \& Kemmelmeier, M. (2002). Rethinking individualism and collectivism: Evaluation of theoretical assumptions and metaanalyses. Psychological Bulletin, 128, 3-72. doi:10. 1037/0033-2909.128.1.3

Pahlke, J., Strasser, S., \& Vieider, F. M. (2015). Responsibility effects in decision making under risk. Journal of Risk Uncertain, 51, 125-146. doi:10.1007/ s1 1166-015-9223-6

Park, T. Y., Kim, S., \& Sung, L. K. (2017). Fair pay dispersion: A regulatory focus theory view. Organizational Behavior and Human Decision Processes, 142, 1-11. doi:10.1016/j.obhdp.2017.07.003

Paterson, I., \& Diekmann, A. (1988). A paradox in decision theory and some experimental results: The relative nature of decisions. Theory and Decision, 25, 107-116. doi:10.1007/bf00134154

Pekerti, A. A., \& Kwantes, C. T. (2011). The effect of self-construals on perceptions of organizational events. International Journal of Cross Cultural Management, 11, 303-323. doi:10.1177/ 1470595811413101

Risse, M. (2004). Arguing for majority rule. Journal of Political Philosophy, 12, 41-64. doi:10.1111/j.14679760.2004.00190.x

Russo, J. E., \& Dosher, B. A. (1983). Strategies for multiattribute binary choice. Journal of Experimental Psychology: Learning, Memory, and Cognition, 9, 676-696. doi:10.1037/0278-7393.9.4.676

Sassenberg, K., Sassenrath, C., \& Fetterman, A. K. (2015). Threat $\neq$ prevention, challenge $\neq$ promotion: The impact of threat, challenge and regulatory focus on attention to negative stimuli. Cognition and Emotion, 29, 188-195. doi:10.1080/02699931. 2014.898612

Saunders, B. (2008). Democracy-as-fairness: Justice, equal chances and lotteries (Doctoral dissertation). University of Oxford.

Shavitt, S., Torelli, C. J., \& Riemer, H. (2011). Horizontal and vertical individualism and collectivism: Implications for understanding psychological processes. In M. Gelfand, C. Y. Chiu, \& Y. Y. Hong (Eds.), Advances in culture and psychology (pp. 309350). New York: Oxford University Press.

Singelis, T. M. (1994). The measurement of independent and interdependent self-construals. Personality and Social Psychology Bulletin, 20, 580-591. doi:10.1177/0146167294205014

Sorkin, R. D., West, R., \& Robinson, D. E. (1998). Group performance depends on the majority rule. Psychological Science, 9, 456-463. doi:10.1111/ 1467-9280.00085 
Summerville, A., \& Roese, N. J. (2008). Self-report measures of individual differences in regulatory focus: A cautionary note. Journal of Research in Personality, 42, 247-254. doi:10.1016/j.jrp.2007.05. 005

Taylor, E., Hewitt, K., Reeves, R. A., Hobbs, S. H., \& Lawless, W. F. (2013). Group decision-making: Consensus rule versus majority rule. Procedia Technology, 9, 498-504. doi:10.1016/j.protcy.2013.12.055

Uskul, A. K., Sherman, D. K., \& Fitzgibbon, J. (2009). The cultural congruency effect: Culture, regulatory focus, and the effectiveness of gain- vs. loss-framed health messages. Journal of Experimental Social Psychology, 45, 535-541. doi:10.1016/j.jesp.2008. 12.005

Wang, J., \& Lee, A. Y. (2006). The role of regulatory focus in preference construction. Journal of Marketing Research, 43, 28-38. doi:10.1509/jmkr.43. 1.28
Wang, X. H., \& Keh, H. T. (2017). Consumer susceptibility to cross-selling persuasion: The roles of selfconstrual and interpersonal harmony. Journal of Retailing and Consumer Services, 34, 177-184. doi:10.1016/j.jretconser.2016.10.008

Yang, H. Y., Stamatogiannakis, A., \& Chattopadhyay, A. (2015). Pursuing attainment versus maintenance goals: The interplay of self-construal and goal type on consumer motivation. Journal of Consumer Research, 42, 93-108. doi:10.1093/jcr/ucv008

Ybarra, O., \& Trafimow, D. (1998). How priming the private self or collective self affects the relative weights of attitudes and subjective norms. Personality and Social Psychology Bulletin, 24, 362-370. doi:10.1177/0146167298244003

Zhang, J., Hsee, C. K., \& Xiao, Z. X. (2006). The majority rule in individual decision making. Organizational Behavior and Human Decision Processes, 99, 102-111. doi:10.1016/j.obhdp.2005.06.004

\section{Appendix}

\section{A Formal Description of the Majority Rule ${ }^{1}$}

Let $\mathcal{A}=\left\{A_{1}, A_{2}, \ldots, A_{n}\right\}$ be a finite set of multidimensional statements.

Let $\mathbb{S}=\{1, \ldots, \mathrm{m}\}$ be a $\mathrm{M}$-dimensional subjective space where the $\mathcal{A}$ is represented, satisfying that $A_{i}$ can and only can be represented as the $i$ th point in the M-dimension space.

The statement $A_{i}$ is characterized on the $\mathrm{M}$ dimensions and is then of the form $A_{i}=\left(\mathrm{O}_{i, 1}, \ldots, \mathrm{O}_{i, m}\right)$, where $\mathrm{O}_{i, j}(j=1, \ldots, \mathrm{m})$ is the objective value level of Statement $A_{i}$ on Dimension $j$. More specifically, either level of Dimension $j$ in the case of Statement $A_{i}$ undergoes a transformation which is a monotonic utility function $u_{i j}=U_{j}^{t, p}\left(\mathrm{O}_{i, j}\right)$ where $p$ is the person who generates the function over a certain time interval $t$, representing the subjective value of $j^{\text {th }}$ component of Statement $A_{i}$ that is estimated by the person. Note that $U_{j}^{t, p}\left(\mathrm{O}_{i, j}\right)$ may be any kind of scale (e.g., nominal, ordinal, interval, or ratio scale values).

$$
\begin{aligned}
& \text { Let } U_{j_{m}}^{t, p}\left(\mathrm{O}_{i_{1}, j_{1}}\right)=\left\{\begin{array}{l}
0 \quad \text { if } U_{j}^{t, p}\left(\mathrm{O}_{i_{1}, j_{1}}\right) \prec_{L} U_{j}^{t, p}\left(\mathrm{O}_{i_{2}, j_{2}}\right), \\
1 \quad \text { if } U_{j}^{t, p}\left(\mathrm{O}_{i_{1}, j_{1}}\right) \succ_{L} U_{j}^{t, p}\left(\mathrm{O}_{i_{2}, j_{2}}\right),^{2}
\end{array} i_{1}, i_{2}=1, \ldots, \mathrm{n}, j_{1}, j_{2}=1, \ldots, \mathrm{m},\right. \\
& \text { and } \\
& U_{j_{s u \mathrm{~m}}}^{t, p}\left(\mathrm{O}_{i, j}\right)=\sum_{i=1}^{n} \Sigma_{j=1}^{m} U_{j_{m}}^{t, p}\left(\mathrm{O}_{i, j}\right), i=1, \ldots, \mathrm{n}, j=1, \ldots, \mathrm{m} .
\end{aligned}
$$

${ }^{1}$ The description is done with the help of Revd Prof. Dr. Marek Porwolik at the Institute of Philosophy, Faculty of Christian Philosophy, Cardinal Stefan Wyszyński University in Warsaw and Prof. Dr. Wim Veys at the Department of Mathematics, Katholieke Universiteit Leuven.

${ }^{2}$ The notations " $\leqslant L$ " and " $>L$ " rather than " $\geqslant$ " and " $>$ " are used to emphasize that the decision maker's judgment may or may not satisfy the utility theory. The notations " $\leqslant$ " and " $>$ " indicate that the axioms are satisfied according to utility theory's definition. 


$$
\begin{aligned}
& \text { Suppose } \\
& \left(\begin{array}{cc}
U_{s u m}^{t, p}\left(\mathrm{O}_{1, j}\right) \\
U_{s u m}^{t, p}\left(\mathrm{O}_{2, j}\right) \\
\ldots \\
U_{s u m}^{t, p}\left(\mathrm{O}_{n, j}\right)
\end{array}\right)=\left(\begin{array}{cc}
\sum_{j=1}^{m} U_{j_{s u m}}^{t, p} & \left(\mathrm{O}_{1, j}\right) \\
\sum_{j=1}^{m} U_{j_{s u m}}^{t, p} & \left(\mathrm{O}_{2, j}\right) \\
\ldots & \\
\sum_{j=1}^{m} U_{j_{s u m}}^{t, p} & \left(\mathrm{O}_{n, j}\right)
\end{array}\right) .
\end{aligned}
$$

If the person employs the majority rule for all $j \in \mathbb{S}$ to compare $A_{i_{1}}$ and $A_{i_{2}}\left(A_{i_{1}}, A_{i_{2}} \in \mathcal{A}, i_{1}, i_{2} \in\{1\right.$, $\ldots, \mathrm{n}\})$, then majority dominance is said to be hold - that is, (1) when $U_{s u m}^{t, p}\left(O_{2, j}\right) \succ_{L} U_{j_{m}}^{t, p}\left(\mathrm{O}_{1, j}\right)$ (i.e., if and only if the total number of preference dominance in which $A_{2}$ is "better than" $A_{1}$ exceeds the total number of preference dominance in which $A_{1}$ is "better than" $A_{2}$ ), $A_{2}$ is majority preferred to $A_{1}$, and (2) when $U_{s u m}^{t, p}\left(O_{2, j}\right)=U_{j m}^{t, p}\left(\mathrm{O}_{1, j}\right)$ (i.e., if and only if the total number of preference dominance in which $A_{2}$ is preferred to $A_{1}$ is equal to the total number of preference dominance in which $A_{1}$ is preferred to $\left.A_{2}\right), A_{i_{2}}$ is equally preferred to $A_{i_{1}}$. 